\title{
EFEITOS DA ADUBAÇÃO NPK NA FERTILIDADE DO SOLO, NUTRIÇÃO E CRESCIMENTO DA SERINGUEIRA ${ }^{(\mathbf{1})}$
}

\author{
O. C. BATAGLIA(2) \& W. R. SANTOS ${ }^{(3)}$
}

\begin{abstract}
RESUMO
O trabalho teve por objetivo avaliar o efeito de diferentes combinações de doses de fertilizantes NPK, durante o período de formação do seringal, sobre as características químicas do solo, nutrição mineral e crescimento de árvores de seringueira [Hevea brasiliensis (Willd. ex Adr. de J uss.) Müell. Arg.], clone RRIM 600. O experimento foi instalado em 1985 e realizado até 1993, no delineamento de blocos ao acaso, num esquema fatorial fracionado $1 / 2$ ( $4 \times 4 \times 4)$, testando as doses anuais de 0, 40, 80 e $120 \mathrm{~kg} \mathrm{ha}^{-1}$ de N, $\mathrm{P}_{2} \mathrm{O}_{5}$ e K $\mathrm{K}_{2} \mathrm{O}$, aplicadas na forma de uréia, superfosfato triplo e cloreto de potássio. Foi usado Latossolo Vermelho-Amarelo Podzólico distrófico A moderado de textura arenosa/média, localizado no município de Avaí, estado de São Paulo, no período entre dois e oito anos de idade das árvores. A avaliação do experimento foi feita por meio da análise de solo, de folha e medida do perímetro do caule. 0 fertilizante nitrogenado (uréia) foi o que cau sou maiores alterações com intensa aci di ficação do solo. Além de alterar diversos atributos do solo, a aplicação de uréia elevou as concentrações de $\mathbf{N}$ e reduziu as de $\mathrm{K}$ e $\mathrm{S}$ nas folhas. A fertilização fosfatada aumentou a disponibilidade de $P$ no solo e a concentração nas folhas, o mesmo acontecendo com a adubação potássica em relação à disponibilidade de $K$ no solo e na planta. Apesar disso, as correlações entre as concentrações de P e K no solo e nas folhas e o período de imaturidade não foram coerentes com esse aumento de disponibilidade. Mesmo sem a aplicação de micronutrientes, observou-se queas concentrações de B nas folhas correlacionaram-se positivamente com o período de imaturidade, enquanto os demais micronutrientes pouco influíram na fertilidade do solo.
\end{abstract}

Termos de indexação: foliar, análise de solo.

(1) Trabal ho parcialmente financiado pelo convênio EMBRAPA/SAA. Recebido para publicação em abril de 1998 e aprovado em setembro de 1999.

(2) Pesquisador Científico do Instituto Agronômico de Campinas - IAC. Caixa Postal 28, CE P 13001-970 Campinas (SP). Bolsista do CNPq.

(3) Engenheiro Agrícola. Bolsista no IAC. 


\title{
SUMMARY: NPK FERTILIZATION EFFECTS ON SOIL FERTILITY, MINERAL NUTRITION AND GROWTH OF RUBBER TREES
}

\begin{abstract}
The purpose of this study was to evaluate soil and plant responses to a continuous supply of NPK fertilizers on rubber trees [Hevea brasiliensis (Willd. ex Adr. de] uss.) Müell. Arg.], clone RRIM 600. The experiment was conducted on a Red-Yellow Podzolic Latosol sand/ medium textureat Avaí, State of SãoPaulo, Brazil. A fractionated factorial experiment 1/ 2 (4 × $4 \times 4$ ) was used, with doses of $0,40,80$ and $120 \mathrm{~kg} \mathrm{ha}^{-1}$ of $\mathrm{N}, \mathrm{P}_{2} \mathrm{O}_{5}$ and $\mathrm{K}_{2} \mathrm{O}$, applied as urea, triple superphosphate and potassium chloride. The evaluations consisted of soil, leaf and trunk girth analyses. The nitrogen fertilizer (urea) promoted the greatest changes in soil fertility, significantly increasing soil acidity. Leaf $\mathrm{N}$ concentration was enhanced while K and S decreased. Phosphate fertilizer increased soil P availability and leaf P. Potassi um fertilizer improved soil and leaf $K$ contents. Leaf $N$ was better correlated with plant growth while soil and leaf $\mathrm{P}$ and $\mathrm{K}$ were not consistently correlated. Borum concentrations in the leaves were positively correlated with the immaturity period of the crop. The availability of other micronutrients was not affected by the fertilizer treatments. Nitrogen and potassium nutrition showed thebest correlations with crop growth. Differences up to twe ve months in theimmaturity period were observed for the different treatments.
\end{abstract}

Index terms: foliar diagnosis, soil analysis.

\section{INTRODUÇÃO}

Em plantações jovens de seringueiras, nas densidades normais com cerca de quinhentas árvores por hectare, o sistema radicular desenvolve-se progressivamente e, se não encontrar limitações, explorará amplamente a profundidade efetiva do solo até o final do quarto ano. Nesse período, a exigência das plantas por água e nutrientes limitase a seu crescimento, sendo parte dos assimilados armazenada na forma de reservas. A necessidade de nutrientes é progressiva, aumentando à medida queas plantas crescem. Fertilizações localizadas na zona de expansão do sistema radicular tendem a acel erar o crescimento das plantas (Watson, 1989).

Boa parte dos conhecimentos sobre os efeitos das adubações na fertilidade dos solos, nutrição e crescimento da seringueira foi gerada nos países orientais. Experimentos realizados em J ava, na década de 30, mostraram respostas de até $29 \%$ a fertilizantes NPK (Dijkman, 1951). Na Malásia, Owen et al. (1957) mostraram comportamentos distintos dos seringais crescendo em solos aluviais costeiros, onde apenas $\mathrm{N}$ era requerido, ao contrário desolos do interior, onde $\mathrm{N}$ era efetivo apenas quando aplicado junto com P e K. Outros experimentos, realizados principalmente na Malásia nas décadas de 60 e 70, revelaram tendências gerais de respostas positivas à fertilização, porém com grande efeitolocal de variações edáficas e mesmo de tratos culturais como a presença ou ausência de leguminosas de cobertura (Watson, 1989).

No Brasil, até à época de instalação deste experimento, em 1985, havia poucos resultados experimentais, a maioria nas regiões norte e nordeste do País. Reis et al. (1984a) observaram reduções dos teores de $K$ nas fol has de plantas em tratamentos com calagem e aumentos dos teores de Ca. Em outro experimento com NPK, Reis et al. (1984b) observaram redução na disponibilidade de Ca eMg no sol o em função da fertilização nitrogenada, comportamento inverso acontecendo com as fertilizações fosfatadas e potássicas. Aplicações de uréia e sulfato de amônio ocasionaram redução nos valores de $\mathrm{pH}$ e aumento de Al trocável (Reis \& Cabala-Rosand, 1988). Fertilizações fosfatadas promoveram aumentos de $\mathrm{P}$ disponível, enquanto as potássicas não alteraram a disponibilidade de $\mathrm{K}$ trocável.

Nos estudos realizados no País, poucas vezes houve preocupação dos autores em quantificar, com precisão, a conseqüência das adubações na fertilidade do solo e na nutrição das plantas. A maioria dedicou maior esforço em constatar os efeitos dos fertilizantes no crescimento ou produtividade das árvores. Trabalhos como o levantamento da situação nutricional de seringais em produção do estado de São Paulo (Bataglia et al., 1988) evidenciaram que as produtividades mais el evadas foram associadas com níveis mais altos de $\mathrm{N}$ e $\mathrm{K}$ nas fol has. Kitamura (1992) observou significativas variações nas concentrações de N, P, K, Ca e Mg nas fol has em função das fertilizações praticadas. Fal cão (1996) e Murbach (1997) observaram respostas ao potássio no crescimento e na produtividade do cl one PB 235, havendo muita coerência entre o aumento da disponibilidade do nutriente no sol o e nas fol has e o incremento de produtividade das plantas. 
Considerando a grande diversidade de respostas e, portanto, a necessidade de quantificação local dessas respostas para a recomendação mais eficaz defertilizantes, realizou-seeste estudo com o objetivo não só de avaliar as al terações na fertilidade do solo e na nutrição mineral de árvores de seringueira submetidas à aplicação de doses crescentes de fertilizantes NPK por longo período em anos sucessivos, mas também relacionar essas alterações com o crescimento do seringal.

\section{MATE RIAL E MÉTODOS}

O estudo foi realizado num plantio comercial de seringueira, clone RRIM 600, na Fazenda São Sebastião, município deAvaí (SP) (Latitude: 2212' S; Longitude: 49o $18^{\prime}$ W, altitude de $520 \mathrm{~m}$ ), no período entre 1985 e 1993.

O sol o da área experimental foi classificado como L atossolo Vermel ho-Amarel o Podzólico distrófico A moderado de textura arenosa/média, apresentando as seguintes características na camada superficial de $0-20 \mathrm{~cm}$ : $80 \mathrm{~g} \mathrm{~kg}^{-1}$ de argila; $50 \mathrm{~g} \mathrm{~kg}^{-1}$ de silte; $870 \mathrm{~g} \mathrm{~kg}^{-1}$ deareia; $6 \mathrm{~g} \mathrm{dm}^{-3}$ de matéria orgânica; $\mathrm{pH}$ em $\mathrm{CaCl}_{2}$ igual a 4,$9 ; 2 \mathrm{mg} \mathrm{dm}^{-3}$ de $\mathrm{P} ; 37 \%$ de saturação por bases e expressos em $\mathrm{mmol}_{\mathrm{C}} \mathrm{dm}^{-3}, 0,6$ de $\mathrm{K}$; 7 de $\mathrm{Ca}$; 2 de $\mathrm{Mg}$ e 17 de H +Al. A análise granulométrica foi feita usando métodos descritos por Camargo et al. (1986), e as análises químicas, de acordo com os métodos de Raij \& Quaggio (1983).

O clima nolocal édo tipo Cwa dealtitude(Setzer, 1966) com temperatura média anual de $21,5^{\circ} \mathrm{C}$ e preci pitação média anual de 1.250 mm, com estação seca no inverno.

O delineamento experimental foi o de blocos ao acaso, no esquema fatorial fracionado $1 / 2(4 \times 4 \times 4)$, conforme proposto por Colwell (1978), com os nutrientes $\mathrm{N}, \mathrm{P}$ eK sendo aplicados em quatro doses: $0,40,80$ e $120 \mathrm{~kg}^{-1}{ }^{-1}$ deN, $\mathrm{P}_{2} \mathrm{O}_{5}$ eK 2 O. Os fertilizantes usados foram uréia, superfosfato triplo e cloreto de potássio. As 32 parcelas foram dispostas em dois blocos. Cada parcela experimental continha duas linhas com 12 árvores no espaçamento de $7 \times 3 \mathrm{~m}$. Foram consideradas como plantas úteis as oito centrais de cada linha. As duas linhas experimentais foram isoladas por uma linha de árvores com fertilização convencional do agricultor, calculada ano a ano de acordo com a recomendação do Boletim no 209 do I nstituto Agronômico (I AC, 1977). Foram usadas as seguintes doses, de acordo com a idade das plantas: $2^{\circ}$ e $3 \circ$ ano, 25-50-45; 4 ao 6o ano, 40-80-60; 70 ano e seguintes, 80-85-75 kg ha-1 ano-1 de $\mathrm{N}, \mathrm{P}_{2} \mathrm{O}_{5}$ e $\mathrm{K}_{2} \mathrm{O}$, respectivamente.

$O$ plantio do seringal foi feito com mudas enxertadas, em fevereiro de 1984. A primeira aplicação de doses diferenciadas de fertilizantes foi realizada em outubro de 1985, quando as árvores tinham 18 meses de idade, no campo. No verão de
$1985 / 86$, as quantidades aplicadas de fertilizantes foram equivalentes a 2/3 das doses normais dos tratamentos. F oram divididas em duas aplicações: uma em novembro e outra em fevereiro. Nessa época, o agricultor realizou uma aplicação localizada de cal cário em faixas laterais às plantas na dose de $1 \mathrm{~kg}$ por árvore em todo o seringal. A partir do verão de 1986/87, aplicaram-se as doses completas de fertilizantes, sempre divididas em duas aplicações (novembro e fevereiro). A última aplicação foi feita, quando as árvores tinham 96 meses de idade, um ano antes das últimas avaliações. A incorporação dos fertilizantes e corretivo foi feita por gradagens superficiais.

A avaliação do crescimento foi feita pela medida do perímetro do caule a 1,20 m acima do calo de enxertia, em outubro, fevereiro e maio de cada ano. A percentagem de árvores aptas à sangria (PAAS) foi obtida pela relação entre o número de árvores com $45 \mathrm{~cm}$ ou mais de perímetro do caule e o total deárvores decada parcela. O período de imaturidade foi determinado por meio de regressões quetiveram como variável dependente a PAAS e variável independente a idade das árvores. O período de imaturidade foi definido como o tempo necessário para se atingir 50\% de árvores aptas à sangria em cada tratamento.

Durante a fase experimental, foram feitas amostragens de solo na área de aplicação dos fertilizantes, uma em maio de 1986, quando as árvores tinham 27 meses de idade, e outra em junho de 1988, aos 51 meses de idade. As amostras de cada parcela eram compostas de 12 a 15 amostras simples da profundidade de $0-20 \mathrm{~cm}$. A análise de solo foi feita com base nos procedimentos descritos por Raij \& Quaggio (1983).

No verão (fevereiro-março) de cada ano, foram feitas amostragens de fol has para acompanhamento do estado nutricional. Foram col hidas fol has sem pecíolo, na base do último lançamento maduro em ramos expostos ao sol, de árvores com menos de quatro anos de idade. Nas árvores adultas, a amostragem foi realizada nos ramos sombreados, aproveitando-se as folhas da base do último Iançamento, também desprezando-se os pecíol os. Os procedimentos usados seguiram as recomendações de Chapman (1973) e Bataglia et al. (1992). As análises químicas das fol has foram feitas de acordo com os métodos descritos por Bataglia et al. (1983).

A inter pretação dos resultados foi feita mediante análises de variância e cálculo de superfícies de respostas, usando um programa de computador especialmente desenvolvido na Seção de Técnica Experimental e Cálculo do Instituto Agronômico. Além disso, procedeu-se a análises de correlação linear e de regressão linear (Steel \& Torrie, 1960) para avaliação das relações entre os resultados da análise de solo ou da planta e o indicador de desenvolvimento do seringal (período de imaturidade). 


\section{RESULTADOSE DISCUSSÃO}

A amostragem de solo em maio de 1986, quando as árvores tinham 27 meses de idade, refletiu não só o impacto das primeiras fertilizações, como também da aplicação localizada de calcário dolomítico, feita pelo agricultor em todo o seringal, que proporcionou altos valores nas determinações relativas à reação do solo (Quadro 1). Como a amostragem foi realizada logo após as apl icações do cal cário e dos fertilizantes, é possível que os val ores el evados depH , Ca, Mgesaturação por bases tenham sido reflexos dessas práticas, uma vez que não houve tempo suficiente para expressiva absorção dos nutrientes pelas árvores e lixiviação no solo. Nessa amostragem, verificou-se grande efeito das fertilizações, principalmente da nitrogenada, que alterou significativamente a maioria das características químicas do sol o na área amostrada.

A análise de solo realizada em maio de 1988, quandoas árvores tinham 51 meses de idade, revel ou uma situação muito mais próxima das condições originais do solo, comparada à amostragem aos 27 meses (Quadro 1). As árvores já tinham porteetaxa de crescimento suficientes para maior absorção de nutrientes graças à maior extensão do sistema radicular e demanda de nutrientes pela parte aérea.
Por causa disso, os valores da análise de solo refletiram também essa maior interação do sistema planta-solo em adição aos efeitos das reações químicas dos fertilizantes e corretivos que predominavam quando as plantas ainda eram pequenas.

Na amostragem real izada aos 51 meses, os efeitos da aplicação localizada de calcário já haviam desaparecido, possivelmente por incorporação do corretivo ao sol o pelas gradagens, pela dissolução e lixiviação de bases e pel a absorção radicular, afetando a reação do solo. De modo geral, nessa amostragem, todas as parcelas apresentaram maior acidez em relação à amostragem anterior. A fertilização nitrogenada mostrou menor poder de acidificação, sem efeito estatisticamente significativo sobre a perda de bases, à exceção do potássio. A fertilização fosfatada promoveu incrementos significativos apenas nas concentrações de $\mathrm{P}$ e $\mathrm{Mg}$, enquanto a potássica alterou apenas os níveis de $\mathrm{K}$ no solo nas duas amostragens.

As alterações promovidas pelas fertilizações na composi ção do sol o foram semel hantes às observadas em outros experimentos com seringueira (Reis et al., 1984a; Reis \& Cabala-Rosand, 1988) e em outras culturas, como café (Moraes et al., 1976) e citros (Cantarella \& Quaggio, 1997).

\section{Quadro 1. Efeitos das doses de nutrientes nos atributos ${ }^{(1)}$ do solo amostrados aos 27 e 51 meses de idade das árvores}

\begin{tabular}{|c|c|c|c|c|c|c|c|c|c|c|c|c|c|c|c|}
\hline \multirow{2}{*}{ Fertilizante } & \multirow{2}{*}{ Dose } & \multicolumn{2}{|c|}{$\mathrm{pH}$} & \multicolumn{2}{|c|}{$\mathrm{P}$} & \multicolumn{2}{|c|}{$\mathrm{K}$} & \multicolumn{2}{|c|}{$\mathrm{Ca}$} & \multicolumn{2}{|c|}{$\mathrm{Mg}$} & \multicolumn{2}{|c|}{$\mathrm{H}+\mathrm{Al}$} & \multicolumn{2}{|c|}{ V } \\
\hline & & 27 & 51 & 27 & 51 & 27 & 51 & 27 & 51 & 27 & 51 & 27 & 51 & 27 & 51 \\
\hline \multirow{3}{*}{$\mathrm{N}$} & $\mathrm{kg} \mathrm{ha}^{-1}$ & & & \multicolumn{2}{|c|}{$-m g d m^{-3}$} & \multicolumn{8}{|c|}{$-\mathrm{mmol}_{\mathrm{c}} \mathrm{dm}^{-3}$} & \multicolumn{2}{|c|}{ — $\%$} \\
\hline & $\begin{array}{r}0 \\
40 \\
80 \\
120\end{array}$ & $\begin{array}{l}6,4 \\
6,1 \\
5,7 \\
5,3\end{array}$ & $\begin{array}{l}4,7 \\
4,5 \\
4,3 \\
4,3\end{array}$ & $\begin{array}{l}64 \\
38 \\
30 \\
45\end{array}$ & $\begin{array}{l}37 \\
51 \\
49 \\
31\end{array}$ & $\begin{array}{l}3,1 \\
2,1 \\
1,6 \\
1,4\end{array}$ & $\begin{array}{l}2,1 \\
1,9 \\
1,7 \\
1,5\end{array}$ & $\begin{array}{l}51 \\
38 \\
28 \\
23\end{array}$ & $\begin{array}{l}9 \\
8 \\
7 \\
6\end{array}$ & $\begin{array}{l}9 \\
7 \\
5 \\
5\end{array}$ & $\begin{array}{l}2 \\
2 \\
2 \\
1\end{array}$ & $\begin{array}{l}14 \\
15 \\
17 \\
19\end{array}$ & $\begin{array}{l}19 \\
19 \\
20 \\
19\end{array}$ & $\begin{array}{l}77 \\
70 \\
62 \\
55\end{array}$ & $\begin{array}{l}39 \\
35 \\
33 \\
31\end{array}$ \\
\hline & & $\mathbf{N}_{\mathbf{L}}(\mathbf{2}) * *$ & & $\mathbf{N}_{\mathbf{Q}} * *$ & & $\mathbf{N}_{\mathrm{L}} * *$ & $\mathbf{N}_{\mathrm{L}} *$ & $\mathbf{N}_{\mathrm{L}} *$ & & $\mathbf{N}_{\mathrm{L}}{ }^{* *}$ & & $\mathbf{N}_{\mathrm{L}}{ }^{* *}$ & & $\mathbf{N}_{\mathbf{L}} * *$ & \\
\hline \multirow[t]{2}{*}{$\mathrm{P}_{2} \mathrm{O}_{5}$} & $\begin{array}{r}0 \\
40 \\
80 \\
120\end{array}$ & $\begin{array}{l}5,4 \\
5,7 \\
6,0 \\
6,3\end{array}$ & $\begin{array}{l}4,4 \\
4,4 \\
4,4 \\
4,5\end{array}$ & $\begin{array}{l}13 \\
42 \\
59 \\
62\end{array}$ & $\begin{array}{r}9 \\
18 \\
47 \\
94\end{array}$ & $\begin{array}{l}2,4 \\
2,1 \\
1,9 \\
1,8\end{array}$ & $\begin{array}{l}2,4 \\
1,9 \\
1,7 \\
1,6\end{array}$ & $\begin{array}{l}22 \\
31 \\
39 \\
47\end{array}$ & $\begin{array}{l}6 \\
7 \\
8 \\
9\end{array}$ & $\begin{array}{l}5 \\
6 \\
7 \\
8\end{array}$ & $\begin{array}{l}1 \\
2 \\
2 \\
2\end{array}$ & $\begin{array}{l}18 \\
17 \\
16 \\
15\end{array}$ & $\begin{array}{l}19 \\
20 \\
20 \\
18\end{array}$ & $\begin{array}{l}57 \\
64 \\
69 \\
74\end{array}$ & $\begin{array}{l}31 \\
32 \\
35 \\
40\end{array}$ \\
\hline & & $\mathbf{P}_{\mathrm{L}} *$ & & $\mathbf{P}_{\mathbf{L}} * *$ & $\mathbf{P}_{\mathrm{L}} * *$ & & & & & $\mathbf{P}_{\mathbf{L}} *$ & $\mathbf{P}_{\mathrm{L}} *$ & & & $\mathbf{P}_{\mathbf{L}} *$ & \\
\hline \multirow[t]{2}{*}{$\mathrm{K}_{2} \mathrm{O}$} & $\begin{array}{r}0 \\
40 \\
80 \\
120\end{array}$ & $\begin{array}{l}5,6 \\
5,9 \\
6,1 \\
6,0\end{array}$ & $\begin{array}{l}4,4 \\
4,4 \\
4,5 \\
4,6\end{array}$ & $\begin{array}{l}49 \\
49 \\
44 \\
35\end{array}$ & $\begin{array}{l}43 \\
48 \\
45 \\
31\end{array}$ & $\begin{array}{l}0,3 \\
1,5 \\
2,7 \\
3,7\end{array}$ & $\begin{array}{l}0,6 \\
1,6 \\
2,3 \\
2,7\end{array}$ & $\begin{array}{l}26 \\
37 \\
41 \\
36\end{array}$ & $\begin{array}{l}7 \\
7 \\
7 \\
9\end{array}$ & $\begin{array}{l}6 \\
6 \\
7 \\
8\end{array}$ & $\begin{array}{l}2 \\
2 \\
2 \\
2\end{array}$ & $\begin{array}{l}18 \\
16 \\
15 \\
16\end{array}$ & $\begin{array}{l}19 \\
20 \\
20 \\
18\end{array}$ & $\begin{array}{l}58 \\
67 \\
70 \\
68\end{array}$ & $\begin{array}{l}32 \\
32 \\
34 \\
40\end{array}$ \\
\hline & & & & & & $\mathbf{K}_{\mathbf{L}} *$ & $\mathbf{K}_{\mathbf{L}} * *$ & & & & & & & & \\
\hline Média & 5,9 & 4,4 & 44 & 42 & 2,1 & 1,8 & 35 & 7 & 7 & 2 & 16 & 19 & 66 & 35 & \\
\hline C.V. (\%) & 11 & 5 & 64 & 86 & 73 & 48 & 49 & 33 & 43 & 25 & 19 & 7 & 19 & 21 & \\
\hline
\end{tabular}

(1) $\mathrm{pH}$ em $\mathrm{CaCl}_{2}$ P , K, Ca e Mg extraídos com resina de troca iônica e H+Al em solução SMP (Raij \& Quaggio, 1983). (2) $\mathrm{N}_{\mathrm{L}}$, $\mathrm{P}_{\mathrm{L}}$ e $\mathrm{K}_{\mathrm{L}}$ : efeitos lineares de N, P e K; $\mathrm{N}_{\mathrm{Q}}$ : efeito quadrático de N. *, ** Significativos a $5 \%$ e $1 \%$, respectivamente. 
O efeito acidificante da uréia observado principalmente na primeira amostragem do solo pode ser explicado, em parte, pela nitrificação dos íons $\mathrm{NH}_{4}{ }^{+}$formados pela sua hidrólise. Além disso, a lixiviação do $\mathrm{NO}_{3}^{-}$pode arrastar cátions dosistema, elevando a concentração de íons $\mathrm{H}^{+}$no solo (Helyar, 1976). Em menor extensão, é possível que parte da acidificação provocada pela aplicação de uréia seja devida a reações que ocorrem na interface raiz-solo por causa da absorção em excesso de cátions em relação a ânions. Para a manutenção do balanço de cargas durante o processo de absorção, íons $\mathrm{H}^{+}$ derivados da dissociação de ácidos orgânicos de dentro das células são passados para a rizosfera, promovendo acidificação (Hiatt \& Leggett, 1974; Kirkby \& Knight, 1977). Convém lembrar, entretanto, que as observações deste experimento são pontuais tanto sob o aspecto espacial como temporal e, por se tratar de um sistema aberto, processos, tais como o crescimento radicular, as diferenças sazonais de umidade do solo e as variações de temperatura, são pouco quantificados nesse tipo de estudo e certamente contribuíram para afetar a absor ção e alterar as concentrações de nutrientes.

As alterações promovidas pelas fertilizações fosfatadas e potássicas eram previsíveis em relação aos aumentos nas disponibilidade dos nutrientes $P$ eK, respectivamente. A fosfatada, entretanto, alterou a atividade de troca catiônica com aumento nas concentrações de $\mathrm{Ca}$ e $\mathrm{Mg}$, redução de $\mathrm{H}+\mathrm{Al}$ e, em conseqüência, aumento na saturação por bases, princi pal mente na primeira amostragem de sol o aos 27 meses de idade das árvores, quando havia efeito de calagem recente. N essa amostragem, fica difícil explicar o aumento significativo de pH com aplicação do superfosfato triplo, um fertilizante cujo equivalente de acidez ou de alcalinidade pode ser considerado nulo (Tisdale et al., 1985).

A aplicação continuada de doses diferenciadas de fertilizantes promoveu respostas distintas de crescimento das plantas aos tratamentos, resultando em diferentes períodos de imaturidade (Quadro 2). Entretanto, relacionando a composição do solo com o período de imaturidade (PI) (Quadro 3), verificouse pouca consistência no sentido das correlações. Principalmente nos casos específicos dos teores de P eK nosolo, esperavam-se coeficientes de correlação negativos, isto é, redução do período de imaturidade com o aumento da disponibilidade dos nutrientes no sol o. E ssa observação ocorreu apenas para P aos 51 meses. Aos 27 meses, os coeficientes de correlação foram positivos para $\mathrm{P}$ eK. Mesmo estratificando os resultados do $\mathrm{PI}$, os resultados for am semel hantes, embora os coeficientes de correlação tivessem sido mais el evados.

É possível que essa relativa incoerência seja também devida às interações entre nutrientes que ocorrem nos experimentos em esquema fatorial. Além disso, é preciso considerar que a amostragem de sol o foi feita na área de aplicação dos fertilizantes, local que abriga apenas parte do sistema radicular. Isso implica na necessidade de reavaliação dos procedimentos de amostragem de solo ou mesmo do modo de aplicação de fertilizantes usualmente recomendados em faixas laterais às árvores (Reis et al., 1982). Um sistema de aplicação em área total

\section{Quadro 2. Períodos de I maturidade (PI) esti mados pela superfície de resposta calculada em função dos} níveis $^{(1)}$ de NPK

\begin{tabular}{|c|c|c|c|c|c|c|c|c|c|c|c|c|c|c|c|}
\hline $\mathbf{N}$ & $\mathbf{P}$ & $K$ & $\mathbf{P I}^{(2)}$ & $\mathbf{N}$ & $\mathbf{P}$ & $K$ & $\mathbf{P I}$ & $\mathbf{N}$ & $\mathbf{P}$ & $\mathbf{K}$ & PI & $\mathbf{N}$ & $\mathbf{P}$ & $K$ & PI \\
\hline & & & meses & & & & meses & & & & meses & & & & meses \\
\hline 1 & 1 & 1 & 81,6 & 2 & 1 & 1 & 80,0 & 3 & 1 & 1 & 80,7 & 4 & 1 & 1 & 83,5 \\
\hline 1 & 1 & 2 & 83,1 & 2 & 1 & 2 & 80,3 & 3 & 1 & 2 & 79,6 & 4 & 1 & 2 & 81,1 \\
\hline 1 & 1 & 3 & 83,7 & 2 & 1 & 3 & 79,6 & 3 & 1 & 3 & 77,6 & 4 & 1 & 3 & 77,9 \\
\hline 1 & 1 & 4 & 83,4 & 2 & 1 & 4 & 77,9 & 3 & 1 & 4 & 74,7 & 4 & 1 & 4 & 73,6 \\
\hline 1 & 2 & 1 & 81,0 & 2 & 2 & 1 & 79,3 & 3 & 2 & 1 & 79,8 & 4 & 2 & 1 & 82,4 \\
\hline 1 & 2 & 2 & 83,1 & 2 & 2 & 2 & 80,1 & 3 & 2 & 2 & 79,3 & 4 & 2 & 2 & 80,6 \\
\hline 1 & 2 & 3 & 84,3 & 2 & 2 & 3 & 80,0 & 3 & 2 & 3 & 77,9 & 4 & 2 & 3 & 77,9 \\
\hline 1 & 2 & 4 & 84,5 & 2 & 2 & 4 & 78,9 & 3 & 2 & 4 & 75,5 & 4 & 2 & 4 & 74,3 \\
\hline 1 & 3 & 1 & 79,5 & 2 & 3 & 1 & 77,7 & 3 & 3 & 1 & 77,9 & 4 & 3 & 1 & 80,4 \\
\hline 1 & 3 & 2 & 82,2 & 2 & 3 & 2 & 79,0 & 3 & 3 & 2 & 78,0 & 4 & 3 & 2 & 79,2 \\
\hline 1 & 3 & 3 & 83,9 & 2 & 3 & 3 & 79,5 & 3 & 3 & 3 & 77,1 & 4 & 3 & 3 & 77,0 \\
\hline 1 & 3 & 4 & 84,7 & 2 & 3 & 4 & 79,0 & 3 & 3 & 4 & 75,4 & 4 & 3 & 4 & 73,9 \\
\hline 1 & 4 & 1 & 77,1 & 2 & 4 & 1 & 75,0 & 3 & 4 & 1 & 75,1 & 4 & 4 & 1 & 77,4 \\
\hline 1 & 4 & 2 & 80,3 & 2 & 4 & 2 & 77,0 & 3 & 4 & 2 & 75,8 & 4 & 4 & 2 & 76,8 \\
\hline 1 & 4 & 3 & 82,6 & 2 & 4 & 3 & 78,0 & 3 & 4 & 3 & 75,5 & 4 & 4 & 3 & 75,2 \\
\hline 1 & 4 & 4 & 84,0 & 2 & 4 & 4 & 78,1 & 3 & 4 & 4 & 74,3 & 4 & 4 & 4 & 72,7 \\
\hline
\end{tabular}

(1) Níveis 1,2,3 e 4 de N, P e K equivalem às doses de $0,40,80$ e $120 \mathrm{~kg} \mathrm{ha}^{-1}$ de $\mathrm{N}, \mathrm{P}_{2} \mathrm{O}_{5}$ e $\mathrm{K}_{2} \mathrm{O}$, respectivamente. ${ }^{(2)} \mathrm{Val}$ ores de $\mathrm{PI}$ calculados pela superfície de resposta: $\mathrm{PI}=-0,065 \mathrm{~N}+0,00068 \mathrm{~N}^{2}-0,002 \mathrm{P}-0,00030 \mathrm{P}^{2}+0,050 \mathrm{~K}-0,00029 \mathrm{~K}^{2}-0,00011 \mathrm{NP}-$ $0,00081 \mathrm{NK}+0,00035 \mathrm{PK}+81,56$. 
Quadro 3. Coeficientes de correlação entre período de imaturidade (PI) e as determinações na análise de solo para amostras individuaís de todas as parcelas (A) e dados médios de nove faixas de PI (B) $)^{(1)}$

\begin{tabular}{|c|c|c|c|c|c|c|c|c|c|}
\hline I dade da árvore & Amostra & MO & pH & $\mathbf{P}$ & $\mathbf{K}$ & $\mathrm{Ca}$ & Mg & $\mathbf{H}+\mathbf{A l}$ & $\mathbf{v}$ \\
\hline \multicolumn{10}{|l|}{ meses } \\
\hline \multirow{2}{*}{27} & A & $-0,63^{*}$ & $0,01^{\mathrm{ns}}$ & $0,73^{* *}$ & $0,40^{\text {ns }}$ & $0,59^{\text {ns }}$ & $0,62^{*}$ & $0,07^{\text {ns }}$ & $-0,02^{n s}$ \\
\hline & B & $0,52 * *$ & $0,34 * *$ & $-0,39 * *$ & $0,12^{\text {ns }}$ & $-0,47 * *$ & $0,66^{* *}$ & $0,02^{\mathrm{ns}}$ & $-0,04^{\text {ns }}$ \\
\hline \multirow{2}{*}{51} & A & $0,07^{\text {ns }}$ & $-0,19^{\text {ns }}$ & $-0,54$ & $0,12^{\text {ns }}$ & $-0,51^{\mathrm{ns}}$ & $-0,37^{\text {ns }}$ & $0,78^{* *}$ & $-0,54^{\text {ns }}$ \\
\hline & B & $0,21^{\text {ns }}$ & $0,29 *$ & $-0,17$ & $0,50 * *$ & $-0,12^{\text {ns }}$ & $0,13^{\text {ns }}$ & $0,48 * *$ & $-0,08^{\text {ns }}$ \\
\hline
\end{tabular}

(1) Faixas de PI: 1-<73; 2-73,0 a 74,4; 3-74,5 a 75,9; 4-76,0 a 77,4; 5-77,5 a 78,9; 6-79,0 a 80,4; 7-80,5 a 81,9; 8-82,0 a 83,4 e 9- >83,4 meses. $*, * *$, ns Significativos a $5,1 \%$ e não-significativo, respectivamente.

nos seringais adultos, visandoaomelhor aproveitamento de nutrientes pelas árvores e maior eficiência da análise de solo, pode ser uma opção mais adequada.

No quadro 4, são apresentados os teores foliares médios apenas para os macronutrientes alterados significativamente pelas diferentes doses dos fertilizantes usadas no experimento. A análise de variância para os teores de macronutrientes nas folhas evidenciou marcante efeito da fertilização nitrogenada na nutrição da seringueira. Em quatro das seis mensurações, houve efeito linear significativo nas concentrações de $\mathrm{N}$ nas folhas. Nessas quatro observações (Quadro 4), houve aumento de $\mathrm{N}$ nas folhas em função das doses crescentes de fertilizante aplicado. A fertilização nitrogenada teve também grande efeito sobre os níveis de $\mathrm{K}$ e $\mathrm{S}$ nas fol has. Na maioria das observações, houve decréscimo do teor deK nas doses mais altas de $\mathrm{N}$. A penas aos 60 meses de idade, $\mathrm{O}$ comportamento foi invertido.

O S foi outro nutriente bastante afetado pela fertilização nitrogenada com comportamento semelhante ao do potássio, isto é, decréscimo nos teores em doses mais altas de N, possivelmente pelo efeito de diluição verificado por causa do maior crescimento das árvores, promovido pela fertilização nitrogenada. Nada confirma, entretanto, uma dúvida inicial sobre possíveis limitações de crescimento pela falta do enxofre nas fertilizações. Os teores deS nas fol has, principalmentea partir dos 36 meses de idade das árvores, mostraram-seacima daquel es observados por Bataglia et al. (1988) em seringais produtivos. Valores muito semelhantes foram observados por Falcão (1996) e Murbach (1997), os quais, mesmo aplicando superfosfato simples em doses crescentes, não verificaram alterações significativas nos teores deS nas árvores, o queevidencia a capaci dadedosolo em suprir essa necessidade.

A fertilização fosfatada e a potássica alteraram pouco o estado nutricional dos demais nutrientes, exceto para os respectivos nutrientes $P$, no caso da adubação fosfatada, e K, na potássica. Para esses nutrientes, houve aumento da concentração nas folhas, à medida que se elevaram as doses dos fertilizantes. Para $P$, as respostas foram mais consistentes, revelando efeitolinear significativo em quatro das seis épocas amostradas e, para o K, em apenas duas épocas. Para a fertilização fosfatada (Quadro 4), os efeitos significativos foram consistentes após os 48 meses de idade das árvores.

Retomando o exame dos efeitos dos tratamentos sobre o período de imaturidade das árvores (Quadro 2), observaram-se grandes variações dePI , desde o menor valor (72,7 meses para o tratamento $444)$ até o mai or (84,5 meses para o tratamento 134), significando praticamente um ano de diferença no início da exploração econômica do seringal, o que é muito expressivo sob o aspecto prático. Verificou-se que, na ausência de aplicação de fertilizantes (111), o período de imaturidade foi menor que em outros tratamentos, mostrando que o desequilíbrio nutricional pode ser mais danoso do que a própria falta de fertilização. Na ausência de fertilização nitrogenada, houve efeito depressivo do fertilizante potássico com conseqüente aumento no período de imaturidade, enquanto, na presença de fertilizante nitrogenado, a situação se inverteu, isto é, o K reduzia o período de imaturidade, à medida que era fornecido em doses mais el evadas.

Como foi discutido anteriormente, as análises de solo tiveram pouca correlação com essas diferenças relatadas. Observando o aspecto da nutrição das plantas, teoricamente, esperavam-se correlações negativas pelo menos entre os teores de $\mathrm{N}, \mathrm{P}$ e K nas fol has eo período de imaturidade, isto é, mel hor nutrição, ocasionando maior crescimento e precocidade do seringal. Nesse aspecto, verificou-se que o teor foliar de $\mathrm{N}$ foi o que melhor se correlacionou com o crescimento do seringal (Quadro 5). Para P e K, os resultados foram inconsistentes. Foram observados coeficientes de correlação negativos na amostragem aos 60 meses, de idade das árvores e positivos aos 72 meses. $\mathrm{Na}$ amostragem feita aos 60 meses, evidenciaram-se os efeitos dos nutrientes N , K, Ca e Mg com cor relações negativas significativas e de $B$ com coeficientes de 
Quadro 4. Teor foliar médio de macronutrientes para cada dose de fertilizante no período compreendido entre os 24 e 84 meses de idade das árvores e valores de teste F para os efeitos linear e quadrático dos tratamentos

\begin{tabular}{|c|c|c|c|c|c|c|c|c|}
\hline \multicolumn{2}{|c|}{ Nutriente } & \multirow{2}{*}{ Idade da árvore } & \multicolumn{4}{|c|}{ Dose do nutriente no fertilizante (kg ha-1) } & \multicolumn{2}{|c|}{ Teste F } \\
\hline No fertilizante & Na folha & & $\mathbf{0}$ & 40 & 80 & 120 & Linear & Quadrático \\
\hline \multirow{4}{*}{$\mathbf{N}$} & & meses & & - & & & & \\
\hline & $\mathrm{N}$ & $\begin{array}{l}24 \\
36 \\
48 \\
60 \\
72 \\
84\end{array}$ & $\begin{array}{l}35,8 \\
31,4 \\
30,4 \\
35,0 \\
27,6 \\
32,5\end{array}$ & $\begin{array}{l}38,1 \\
32,8 \\
29,3 \\
35,6 \\
28,2 \\
31,3\end{array}$ & $\begin{array}{l}39,3 \\
33,9 \\
29,9 \\
37,0 \\
29,3 \\
31,3\end{array}$ & $\begin{array}{l}39,4 \\
34,7 \\
32,0 \\
39,1 \\
30,7 \\
32,4\end{array}$ & $\begin{array}{c}8,47 * * \\
11,95^{* *} \\
2,13 \\
16,95 * * \\
23,74 * * \\
0,01\end{array}$ & $\begin{array}{l}1,45 \\
0,22 \\
3,64 \\
1,07 \\
0,63 \\
2,89\end{array}$ \\
\hline & K & $\begin{array}{l}24 \\
36 \\
48 \\
60 \\
72 \\
84\end{array}$ & $\begin{array}{r}10,8 \\
13,8 \\
9,0 \\
10,7 \\
10,1 \\
14,7\end{array}$ & $\begin{array}{r}10,4 \\
12,6 \\
8,4 \\
11,1 \\
9,8 \\
13,0\end{array}$ & $\begin{array}{r}10,3 \\
12,0 \\
8,0 \\
11,9 \\
9,2 \\
12,5\end{array}$ & $\begin{array}{r}10,5 \\
11,9 \\
7,9 \\
12,9 \\
8,0 \\
13,3\end{array}$ & $\begin{array}{c}1,10 \\
17,50^{* *} \\
4,90^{*} \\
21,68^{* *} \\
13,37 * * \\
4,67 *\end{array}$ & $\begin{array}{l}1,52 \\
2,50 \\
0,43 \\
0,70 \\
1,23 \\
6,61^{*}\end{array}$ \\
\hline & $\mathrm{s}$ & $\begin{array}{l}24 \\
36 \\
48 \\
60 \\
72 \\
84\end{array}$ & $\begin{array}{l}1,7 \\
2,8 \\
2,2 \\
2,0 \\
3,6 \\
2,5\end{array}$ & $\begin{array}{l}1,6 \\
2,7 \\
2,0 \\
1,8 \\
3,2 \\
2,4\end{array}$ & $\begin{array}{l}1,6 \\
2,8 \\
1,8 \\
1,8 \\
2,6 \\
2,3\end{array}$ & $\begin{array}{l}1,6 \\
3,0 \\
1,7 \\
2,0 \\
2,0 \\
2,3\end{array}$ & $\begin{array}{c}6,21^{*} \\
1,72 \\
13,46^{* *} \\
0,01 \\
207,63^{* *} \\
4,46^{*}\end{array}$ & $\begin{array}{l}1,46 \\
1,36 \\
0,32 \\
5,10^{*} \\
1,69 \\
0,39\end{array}$ \\
\hline $\mathbf{P}_{2} \mathrm{O}_{5}$ & $P$ & $\begin{array}{l}24 \\
36 \\
48 \\
60 \\
72 \\
84\end{array}$ & $\begin{array}{l}1,8 \\
1,9 \\
1,8 \\
2,0 \\
1,6 \\
1,9\end{array}$ & $\begin{array}{l}1,8 \\
2,0 \\
1,8 \\
2,2 \\
1,8 \\
2,2\end{array}$ & $\begin{array}{l}1,8 \\
2,0 \\
1,9 \\
2,3 \\
2,0 \\
2,4\end{array}$ & $\begin{array}{l}1,7 \\
2,0 \\
1,9 \\
2,3 \\
2,1 \\
2,5\end{array}$ & $\begin{array}{c}2,56 \\
0,31 \\
5,08^{*} \\
9,92 * * \\
37,08^{* *} \\
32,10^{* *}\end{array}$ & $\begin{array}{l}0,03 \\
0,27 \\
0,28 \\
3,24 \\
0,99 \\
1,03\end{array}$ \\
\hline $\mathrm{K}_{2} \mathrm{O}$ & $\mathrm{K}$ & $\begin{array}{l}24 \\
36 \\
48 \\
60 \\
72 \\
84\end{array}$ & $\begin{array}{r}10,1 \\
11,6 \\
8,0 \\
11,0 \\
8,8 \\
12,5\end{array}$ & $\begin{array}{r}10,5 \\
12,8 \\
8,6 \\
11,9 \\
9,5 \\
13,2\end{array}$ & $\begin{array}{r}10,7 \\
13,1 \\
8,6 \\
12,1 \\
9,6 \\
13,7\end{array}$ & $\begin{array}{r}10,8 \\
12,7 \\
8,1 \\
11,6 \\
9,2 \\
14,1\end{array}$ & $\begin{array}{l}4,18 \\
5,92^{*} \\
0,07 \\
1,26 \\
0,51 \\
5,77^{*}\end{array}$ & $\begin{array}{l}0,38 \\
5,44^{*} \\
2,06 \\
3,28 \\
2,08 \\
0,07\end{array}$ \\
\hline
\end{tabular}

*,** Significativos a 5 e $1 \%$, respectivamente.

correlação positivos, indicando que, mesmo sem a adição desse micronutriente, o solo supriu as necessidades da planta.

O agrupamento dos tratamentos em classes de período de imaturidade (Quadro 6) melhorou a interpretação das relações entre os teores de nutrientes e o crescimento das plantas Verificou-se que os coeficientes de correlação foram, na maioria dos casos, mais el evados (Quadro 5). O mesmo pode ser verificado na figura 1 onde foram plotados os teores de N, P eK nas fol has aos 60 meses de idade das árvores eos respectivos períodos de imaturidade. Quando os dados de PI foram estratificados, a interpretação para N e K melhorou sensivelmente, enquanto para $\mathrm{P}$ isso não aconteceu.

As respostas observadas às fertilizações (nitrogenada e potássica) quanto ao crescimento e precocidade do seringal são fundamentadas nas funções desempenhadas por esses nutrientes na planta. É conhecido para a seringueira (Shorrocks, 1979) que o maior suprimento de $\mathrm{N}$ resulta em maior conteúdo de proteínas, sendo o processo ativado pelo suprimento de K. Além de atuar no processo de síntese, evitando acúmulo de compostos nitrogenados solúveis, o $\mathrm{K}$ atua também na translocação de assimilados para as áreas de crescimento, favorecendo a expansão foliar e, em conseqüência, o crescimento da planta (Marschner, 1997). Por outro lado, mesmo com as limitações observadas sobre as correlações entre concentração foliar e crescimento observadas para $P$, esse nutriente desempenha papel relevante em reações bioquímicas do metabolismo dos carboidratos, particularmente nos processos da respiração, da 
divisão celular e no desenvolvimento dos tecidos meristemáticos. Talvez por essas razões os nutrientes $\mathrm{N}, \mathrm{P}$ eK sejam freqüentemente citados como os mais envolvidos no crescimento das seringueiras (Bataglia et al., 1988), sendo muito exigidos pela planta durante o crescimento (Shorrocks, 1965), uma vez que, durante a fase produtiva num seringal estabilizado, as quantidades de nutrientes exportadas com a produção de látex são muito pequenas (Haag et al., 1990; Murbach, 1997).

Quadro 5. Coeficientes de correlação entre período de imaturidade (PI) e os teores foliares para parcelas individuais (A) e dados médios de nove faixas de PI (B) ${ }^{(1)}$, aos 60 e 72 meses de idade das árvores

\begin{tabular}{|c|c|c|c|c|}
\hline \multirow{3}{*}{ Nutriente } & \multicolumn{4}{|c|}{ Idade (meses) } \\
\hline & \multicolumn{2}{|c|}{60} & \multicolumn{2}{|c|}{72} \\
\hline & $\mathbf{A}$ & B & $\mathbf{A}$ & B \\
\hline $\mathrm{N}$ & $-0,61 * *$ & $-0,93 * *$ & $-0,37 * *$ & $-0,89 * *$ \\
\hline$P$ & $-0,21$ & $-0,40$ & $0,51^{* *}$ & $0,90 * *$ \\
\hline $\mathrm{K}$ & $-0,55^{* *}$ & $-0,94 * *$ & $0,52^{* *}$ & $0,85 * *$ \\
\hline $\mathrm{Ca}$ & $-0,52 * *$ & $-0,71^{*}$ & 0,21 & $0,65 *$ \\
\hline $\mathrm{Mg}$ & $-0,63 * *$ & $-0,89 * *$ & 0,05 & 0,46 \\
\hline S & 0,10 & 0,10 & $0,60 * *$ & $0,97 * *$ \\
\hline $\mathrm{B}$ & $0,66 * *$ & $0,85^{* *}$ & $0,66 * *$ & 0,63 \\
\hline $\mathrm{Cu}$ & 0,13 & $-0,09$ & $-0,30 *$ & $-0,41$ \\
\hline $\mathrm{Fe}$ & $-0,21$ & 0,03 & $-0,46 * *$ & $-0,76^{*}$ \\
\hline $\mathrm{Mn}$ & $-0,13$ & 0,10 & $-0,53 * *$ & $-0,90 * *$ \\
\hline $\mathrm{Zn}$ & $-0,10$ & $-0,53$ & $0,40 * *$ & $0,70^{*}$ \\
\hline
\end{tabular}

(1) Faixas de PI: 1 - < 73; 2 -73,0 a 74,4; 3-74,5 a 75,9; 4-76,0 a 77,4; 5-77,5 a 78,9; 6-79,0 a 80,4; 7-80,5 a 81,9; 8-82,0 a 83,4 e 9$>83,4$ meses. $*, * *$ Significativos a 5 e $1 \%$, respectivamente.

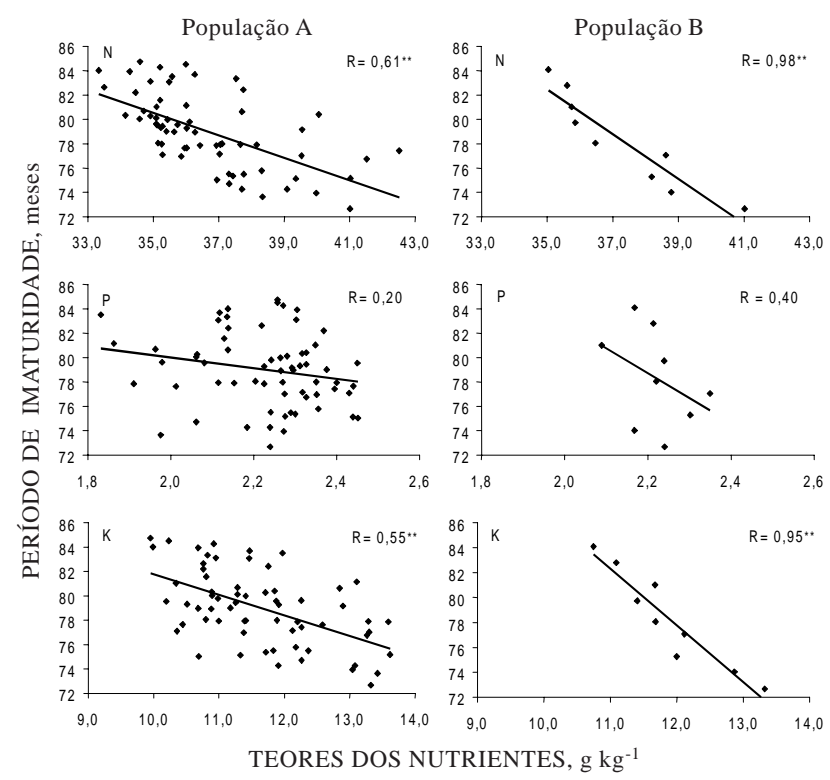

Figura 1. Relação entre teores foliares e período de imaturidade de seringueiras para dados individuais de todas as parcelas (População A) e média de nove estratos (População B).

Quadro 6. Teores foliares médios das nove classes de período de imaturidade (PI), em amostragens de folhas, aos 60 e 72 meses de idade das árvores

\begin{tabular}{|c|c|c|c|c|c|c|c|c|c|c|c|c|}
\hline Faixa de PI & PI médio & $\mathbf{N}$ & $\mathbf{P}$ & $\mathbf{K}$ & $\mathbf{C a}$ & Mg & $\mathbf{S}$ & B & $\mathbf{C u}$ & $\mathbf{F e}$ & Mn & $\mathbf{Z n}$ \\
\hline \multicolumn{2}{|c|}{ meses } & \multicolumn{6}{|c|}{$\longrightarrow \mathrm{g} \mathrm{kg}^{-1}$} & \multicolumn{5}{|c|}{$-\mathrm{mg} \mathrm{kg}^{-1}$} \\
\hline \multicolumn{13}{|c|}{ Amostragem aos 60 meses } \\
\hline$<73$ & 72,7 & 41,0 & 2,2 & 13,3 & 13,6 & 3,7 & 2,0 & 34 & 14 & 116 & 256 & 30 \\
\hline 73,0 a 74,4 & 74,0 & 38,8 & 2,2 & 12,9 & 13,8 & 3,6 & 1,9 & 33 & 13 & 123 & 254 & 27 \\
\hline 74,5 a 75,9 & 75,3 & 38,2 & 2,3 & 12,0 & 14,2 & 3,6 & 1,9 & 33 & 11 & 140 & 287 & 26 \\
\hline 76,0 a 77,4 & 77,1 & 38,6 & 2,3 & 12,1 & 14,0 & 3,7 & 2,0 & 36 & 13 & 130 & 280 & 27 \\
\hline 77,5 a 78,9 & 78,1 & 36,5 & 2,2 & 11,7 & 13,9 & 3,4 & 1,8 & 34 & 11 & 139 & 280 & 26 \\
\hline 79,0 a 80,4 & 79,7 & 35,9 & 2,2 & 11,4 & 13,9 & 3,5 & 1,9 & 35 & 11 & 140 & 288 & 25 \\
\hline 80,5 a 81,9 & 81,0 & 35,7 & 2,1 & 11,7 & 13,2 & 3,4 & 1,9 & 36 & 12 & 128 & 281 & 26 \\
\hline 82,0 a 83,4 & 82,8 & 35,6 & 2,2 & 11,1 & 13,0 & 3,2 & 2,0 & 40 & 13 & 124 & 271 & 27 \\
\hline$>83,4$ & 84,1 & 35,0 & 2,2 & 10,7 & 12,7 & 2,9 & 1,9 & 40 & 14 & 121 & 254 & 27 \\
\hline \multicolumn{13}{|c|}{ Amostragem aos 72 meses } \\
\hline$<73$ & 72,7 & 29,5 & 1,7 & 7,1 & 13,0 & 2,6 & 1,9 & 43 & 8 & 146 & 381 & 25 \\
\hline 73,0 a 74,4 & 74,0 & 29,7 & 1,9 & 8,0 & 15,1 & 2,8 & 2,1 & 38 & 9 & 135 & 366 & 23 \\
\hline 74,5 a 75,9 & 75,3 & 29,5 & 2,4 & 8,9 & 16,2 & 3,0 & 2,6 & 36 & 9 & 132 & 336 & 24 \\
\hline 76,0 a 77,4 & 77,1 & 29,8 & 2,5 & 8,8 & 15,6 & 3,0 & 2,5 & 38 & 9 & 130 & 327 & 25 \\
\hline 77,5 a 78,9 & 78,1 & 29,1 & 2,6 & 9,5 & 17,3 & 3,1 & 2,8 & 38 & 8 & 129 & 336 & 24 \\
\hline 79,0 a 80,4 & 79,7 & 28,6 & 2,7 & 9,5 & 17,6 & 3,3 & 3,0 & 41 & 8 & 130 & 326 & 25 \\
\hline 80,5 a 81,9 & 81,0 & 28,5 & 2,4 & 8,7 & 16,1 & 3,1 & 2,9 & 44 & 9 & 135 & 333 & 25 \\
\hline 82,0 a 83,4 & 82,8 & 28,3 & 3,0 & 9,8 & 16,2 & 3,0 & 3,3 & 44 & 8 & 127 & 306 & 26 \\
\hline$>83,4$ & 84,1 & 28,4 & 3,1 & 10,0 & 16,7 & 2,8 & 3,3 & 45 & 7 & 124 & 305 & 25 \\
\hline
\end{tabular}




\section{CONCLUSÃO}

1. A aplicação continuada de doses diferenciadas de fertilizantes promoveu respostas distintas no crescimento das plantas de seringueira, evidenciadas por diferenças de até doze meses nos períodos de imaturidade das árvores.

2. A análise de sol o permitiu avaliar os efeitos de acidificação, provocados pela aplicação contínua de uréia, e o aumento na disponibilidade de $\mathrm{P}$ e $\mathrm{K}$, provocado pelas adubações fosfatada e potássica, mas mostrou-se pouco eficiente para quantificar as relações entre a disponibilidade no sol o e o período de imaturidade das árvores.

3. As mel hores correlações foram observadas entre os teores de $\mathrm{N}$ nas fol has e o período de imaturidade. Para P eK, não houve consistência das correlações nas diferentes épocas de amostragem Mesmo sem aplicação de S e de micronutrientes, o solo supriu adequadamente as necessidades das plantas para esses nutrientes.

\section{AGRADE CIMENTOS}

Ao Dr. J ayme Vasques Cortez, pela cooperação na instalação do experimento. Aos pesquisadores Toshio I gue, Paulo de Souza Gonçalves e Mário Cardoso, pela cooperação no planejamento e condução do experimento. À F azenda São Sebastião, pel o apoi o no trabal ho experimental, eà EMBRAPA, pel o financiamento parcial da pesquisa por meio do Convênio EMBRAPA/SAA.

\section{LITERATURA CITADA}

BATAGLIA, O.C.; CARDOSO, M. \& CARRETERO, M.V. Situação nutricional de seringais produtivos no estado de São Paulo. Bragantia, 47:109-123, 1988.

BATAGLIA, O.C.; DECHEN, A.R. \& SANTOS, W.R. Diagnose visual eanálise de plantas. In: DECHEN, A.R.; BOARETTO, A.E. \& VERDADE, F.C., eds. Adubação, produtividade e ecologia. Campinas, Fundação Cargill, 1992. p.369-393.

BATAGLIA, O.C.; FURLANI, A.M.C.; TEIXEIRA, J .P.F.; FURLANI, P.R. \& GALLO,J .R. Métodos deanálisequímica de plantas. Campinas, Instituto Agronômico de Campinas, 1983. 48p. (Boletim Técnico, 78)

CAMARGO, O.A.; MONIZ, A.C.; J ORGE, J .A. \& VALADARES, J .M.A.S. Métodos de análise química e física de solos do Instituto Agronômico de Campinas. Campinas, Instituto Agronômico de Campinas, 1986. 94 p. (Boletim Técnico, 106)

CANTARELLA, H. \& QUAGGIO, J.A. Eficiência de adubação nitrogenada em citros. Campinas, I nstituto Agronômico de Campinas, 1997. 14p. (Relatório Fazenda Cambuhy)

CHAPMAN, H.D. Diagnostic criteria for plants and soils. Riverside, H.D. Chapman, 1973. 793p.
COLWELL, J.D. Computations for studies of soil fertility and fertilizer requirements. Slough, Commonw. Agric. Bur., 1978. 297p.

DIJ KMAN, M.I. Hevea: Thirty years of research in the Far East. Miami, University of Miami Press, 1951. 236p.

FALCÃO, N.P.S. Adubação NPK afetando o desenvolvimento do caule da seringueira e parâmetros fisiológicos do látex. Piracicaba, Escola Superior de Agricultura "Luiz de Queiroz" 1996. 134p. (Tese de Doutorado)

HAAG, H.P.; BUENO, N. \& PEREIRA, J.P. Alguns aspectos recentes da nutrição da seringueira no Brasil. In: SIMPÓSIO DA CULTURA DA SERINGUEIRA, 2. Piracicaba, 1987. Anais. Piracicaba, Escola Superior de Agricultura Luiz de Queiroz, 1990. p.59-88.

HELYAR, K.R. Nitrogen cycling and soil acidification. J .Aust.Inst.Agric.Sci 42:217-221, 1976.

HIATT, A.J . \& LEGETT, J .E. I onic interactions and antagonisms in plants. In: CARSON, E.W., ed. The plant root and its environment. Charlotesville, University Press of Virginia, 1974. p. 101-134.

INSTITUTO AGRONÔMICO DE CAMPINAS - IAC. Tabelas de adubação e de calagem. Campinas, 1977. 198p. (Boletim Técnico, 209)

KIRKBY, E.A. \& KNIGHT, A.H. Influence of the level of nitrate nutrition on ion uptake and assimilation, organic acid accumulation, and cation-anion balance in whole tomato plants. Plant Physiol., 60:349-353, 1977.

KITAMURA, M.C. Influência dos níveis de nitrogênio, fósforo, potássio e magnésio no desenvolvimento de seringueira jovem (Hevea brasiliensis Muell. Arg.) em solo de cerrado de Mato Grosso do Sul. Lavras, Escola Superior de Agricultura de Lavras.1992. 90p. (Tese de Mestrado)

MARSCHNER, H. Mineral nutrition of higher plants. 2.ed. London, Academic Press, 1997. 889p.

MORAES, F.R.P.; LAZZARINI, W.;TOLEDO, S.V.; CERVELLINI, G. \& FUJ IWARA, M.O. Fontes e doses de nitrogênio na adubação química do cafeeiro. Latossolo Roxo, transição para Latossolo Vermelho-Amarelo orto. Bragantia, 35:6377, 1976.

MURBACH, M.R. Efeitos de níveis de nitrogênio, fósforo e potássio no desenvolvimento, produtividade de borracha seca e exportação denutrientes pela seringueira. Piracicaba, Escola Superior de Agricultura "Luiz de Queiroz", 1997. 94p. (Tese de Mestrado)

OWEN, G., WESTGARTH, O. R., IYER, G. C. Manuring Hevea: effects of fertilizers on growth and yield of mature rubber trees. J . Rubber Res. Inst. Malaya, 15:29-52, 1957.

RAIJ , B.van \& QUAGGIO, J .A. Métodos de análise de sol os para fins de fertilidade. Campinas, Instituto Agronômico de Campinas, 1983. 31p. (Boletim Técnico, 81)

REIS, E.L. \& CABALA-ROSAND, P. Eficiência dos fertilizantes aplicados nas fases de pré e pós sangria da seringueira. $R$. Theobroma, 18:189-200, 1988.

REIS, E.L.; CABALA-ROSAND, P \& SANTANA, C.J .L. Indicações de adubação de seringueira no Sul da Bahia. Ilhéus, CEPLAC/SUDHEVEA - Centro de Pesquisas do Cacau, 1982. 16p. 
REIS, E.L.; SANTANA, C.J .L. \& CABALA-ROSAND, P. I nfluência da calagem e adubação na produção da seringueira no sul da Bahia. R. Theobroma, 14:33-44, 1984a.

REIS, E.L.; SOUZA, L.F.S. \& MELLO, F.A.F. Influência da aplicação de nitrogênio, fósforo e potássio sobre o desenvolvimento da seringueira (Hevea brasiliensis Muell. Arg.) no sul da Bahia. R. Theobroma, 14:45-52, 1984b.

SETZER, J . Atlas climático e ecológico do estado de São Paulo. Comissão Interestadual da Bacia Paraná Uruguai. São Paulo, Companhia Energética de São Paulo, 1966. 61p.

SHORROCKS, V.M. Deficiências minerais em Hevea e plantas de cobertura associadas. Brasília, Superintendência da Borracha, 1979. 76p.
SHORROCKS, V.M. Mineral nutrition and nutrient cycle of Hevea brasiliensis. I. Growth and nutrient content. J . Rubber Res. Inst. Malaya, 19:32-47, 1965.

STEEL, R.G.D. \& TORRIE, J.H. Principles and procedures of statistics, with special reference to biological sciences. New York, McGraw Hill Book Company, 1960. 481p.

TISDALE, S.L.; NELSON, W.L. \& BEATON, J .D. Soil Fertility and Fertilizers. 4.ed. New York, Macmillan Publishing Company, 1985. 754p.

WATSON, G.A. Nutrition. In: WEBSTER, C.C. \& BAULKIWILL, W.J ., ed. Rubber. Essex, Longman Scientific and Technical, 1989. p.291-347. 